12

\title{
Увлечение электронов в полупроводниковой наноструктуре потоком нейтральных частиц
}

\author{
(C) С.В. Ганцевич, В.Л. Гуревич \\ Физико-технический институт им. А.Ф. Иофрфе РАН, \\ Санкт-Петербург, Россия \\ E-mail: sergei.elur@mail.ioffe.ru
}

(Поступила в Редакцию 25 июля 2018 г.)

Рассматривается увлечение носителей тока (электронов) в двумерной полупроводниковой наноструктуре при движении вблизи ее поверхности потока нейтральных частиц (атомов или молекул). Показано, что увлечение по физическому механизму похоже увлечению электронов ионным пучком в квантовых проволоках, рассмотренному ранее в работах В.Л. Гуревича и М.И. Мурадова.

DOI: 10.21883/FTT.2019.01.46909.220

\begin{abstract}
Эффект увлечения носителей тока в одной физической системе под влиянием потока частиц в другой системе неоднократно исследовался теоретически и экспериментально (см., например, работы [1-7]). Увлечение носителей тока в наноструктуре ионным пучком рассматривалось в работах $[8,9]$. В общем случае увлечение связано с возможностью обмена импульсом (для кристаллов - квазимпульсом) между двумя взаимодействующими системами. Одна из них - активная система имеет отличный от нуля полный (квази)импульс и может взаимодействовать со второй - пассивной системой с исходным нулевым полным (квази)импульсом. При включении межчастичного взаимодействия импульс активной системы распространится на частицы пассивной системы. Поскольку общий центр инерции систем не может измениться под действием внутренних сил, пассивная система обязана прийти в движение, получив часть импульса активной системы. Всегда имеющаяся диссипация в активной системе компенсируется в стационарном режиме приложенной внешней силой, а диссипация в пассивной системе определяет величину увлечения через баланс приходящего и уходящего импульса.
\end{abstract}

В нашем случае активная система - газ нейтральных атомов или молекул, поток которых охватывает пассивную систему в виде полупроводниковой квазидвумерной наноструктуры с невырожденным электронным газом на низшем уровне поперечного квантования.

Величину увлечения электронов тяжелыми частицами $(M \gg m)$ оценим из следующих соображений. Обе системы в стационарном режиме считаем пространственно однородными, исключая поперечный размер. В поперечном направлении потенциал взаимодействия, создаваемый частицами активной системы, мало меняется в пассивной области из-за сравнительно большого расстояния между ними и электронами наноструктуры и малой толщины пассивной области. Этот потенциал для достаточно тяжелых частиц можно приближенно считать движущейся преградой, упруго отражающей электроны с определенной вероятностью и тем самым передающий им импульс $m V$ в направлении своего движения. При частоте столкновений электронов с преградой $v_{d}=1 / \tau_{d}$ вносимый импульс в среднем равен $m V v_{d}$. В свою очередь электроны наноструктуры из-за рассеяния на примесях и фононах теряют направленную скорость с частотой релаксации $v_{e}=1 / \tau_{e}$.

В стационарном режиме устанавливается баланс между скоростью увлечения $V$ и приобретенной электронами дрейфовой скоростью $V^{\prime}$

$$
\left(V-V^{\prime}\right) v_{d}=V^{\prime} v_{e}, \quad V^{\prime}=V \frac{v_{d}}{v_{e}+v_{d}}=\kappa V
$$

В отсутствие эффективного трения $v_{e} \rightarrow 0$ и при любой величине взаимодействия $v_{d} \neq 0$ в стационарном режиме достигается полное увлечение $V=V^{\prime}$ и передача импульса прекращается. В этом предельном случае полного увлечения в пассивной системе ток будет равен $j^{d}=e n V$, где $n-$ концентрация электронов, а $e-$ их заряд. Для потока бесстолкновительного нейтрального газа, который можно получить фильтрацией теплового источника достаточно высокой температуры $T_{d}$, скорость увлекающего потока может быть порядка $V \simeq \sqrt{T_{d} / M}$, а скорость увлечения соответственно в $1 / \kappa$ раз меньше.

Электрическое поле увлечения $E_{d}$ можно оценить обычным образом через полученную скорость дрейфа электронов $V^{\prime}$ и подвижность носителей тока $\mu=e \tau / m$. Если приравнять полное обратное время релаксации $\tau$ сумме $v_{e}+v_{d}$, то поле увлечения будет зависеть только от частоты рассеяния $v_{d}=1 / \tau_{d}$, которая, таким образом, определяет „подвижность увлечения“ как $\mu_{d}=e \tau_{d} / m$. Эта подвижность позволяет пересчитать скорость увлекающего потока в поле увлечения как $E_{d}=V / \mu_{d}$.

Приведенные выше качественные соображения позволяют грубо оценить величину увлечения через отношение времен релаксации $\nu_{d} / \nu_{e}=\tau / \tau_{d} \simeq V^{\prime} / V=\kappa$. Малость этой величины определяется, в основном, большим расстоянием между носителем тока - электроном и рассеивающей частицей, если принять число рассеивателей внутри наноструктуры порядка концентра- 
ции частиц увлекающего потока и считать примерно одинаковыми механизмы взаимодействия при рассеянии.

Теперь рассмотрим нашу задачу на основе уравнения Больцмана.

Ток увлечения выражается обычным образом через функцию распределения электронов в пассивной области единичного объема (для краткости далее мы не будем явно выписывать векторные значки).

$$
J_{\alpha}^{d}=-e^{2} \sum_{p} v_{\alpha} \Delta F_{p}
$$

где $\Delta F_{p}$ - добавка к функции распределения электронов в пассивной области, вызванная действием потока внешних частиц. Эти частицы создают в пассивной области движущийся возмущающий потенциал. Рассеяние электронов на этом потенциале сопровождается переносом (квази)импульса от внешнего потока частиц в пассивную область, что можно описать изменением функции распределения электронов в пассивной области согласно уравнению Больцмана

$$
\left(\partial_{t}+I_{p}\right) \Delta F_{p}+\Pi_{p}(F)=0 .
$$

Здесь $I_{p}-$ обычный интеграл столкновений электронов с рассеивателями в пассивной области (фононы и примеси), а „оператор увлечения“ $\Pi_{p}(F)$ описывает влияние потока активных внешних частиц. Имеем

$$
I_{p} \Delta F_{p} \equiv \sum_{k}\left(w_{k p} \Delta F_{p}-w_{p k} \Delta F_{k}\right)
$$

Действие внешних частиц также можно расматривать как столкновение

$$
\Pi_{p}\left(F_{p}\right)=\sum_{k l l^{\prime}}\left(W_{k l^{\prime}}^{p l} F_{p} \Phi_{l}-W_{p l}^{k l^{\prime}} F_{k} \Phi_{l^{\prime}}\right) .
$$

Интеграл парных столкновений (5) обращается в ноль равновесными функциями распределения, так что хотя бы одна из функций распределения в этом интеграле должна быть неравновесной. Рассмотрим сначала случай функции распределения внешних частиц с фиксированной скоростью $\Phi \sim \delta(v-V)$, которая неравновесна по определению.

Ввиду малости эффекта увлечения в качестве функции распределения электронов в (4) можно ставить равновесную функцию

$$
F_{p}=F_{p}^{e q}+\Delta F_{p} \simeq F_{p}^{e q}
$$

которую будем считать функцией распределения Больцмана с температурой $T: F_{p}^{e q} \sim \exp \left(-p^{2} / 2 m T\right)$.

Вероятности переходов в операторе столкновений с рассеивателями в пассивной области имеют стандартный вид, поэтому задача состоит в определнии вероятности $W_{p k}^{l l^{\prime}}$ в операторе увлечения (5).

Ограничимся расчетом вероятности $W_{p k}^{l l^{\prime}}$ в борновском приближении, полагая их волновые функции плоскими волнами. Эта вероятность обычным образом выражается через квадрат матричного элемента перехода между состояниями $(p l)$ и $k l^{\prime}$ :

$$
\begin{aligned}
W_{p k}^{l l^{\prime}}= & 2 \pi\left|\left\langle p l\left|U\left(r, r^{\prime}\right)\right| k l^{\prime}\right\rangle\right|^{2} \\
& \times \delta\left(p+l-k-l^{\prime}\right) \delta\left(\epsilon_{p}+\varepsilon_{l}-\epsilon_{k}-\varepsilon_{l^{\prime}}\right) .
\end{aligned}
$$

Потенциал взаимодействия между электронами наноструктуры и нейтральными частицами, имеет сложную форму, зависящую от распределения существующих зарядов в целом нейтральной частицы, а также от зарядов, возникающих вследствие ее поляризации при взаимодействии с электронами наноструктуры.

Для потенциала, создаваемого фиксированным распределением зарядов $e_{j}$ увлекающей частицы, имеем

$$
\begin{aligned}
U(\mathbf{R}) & =\sum_{j} \frac{e_{j}}{\sqrt{\left|\mathbf{R}-\mathbf{r}_{j}\right|}} \\
& =\sum_{j} e_{j} e^{-\mathbf{r}_{j} \nabla} \frac{1}{R}=\sum_{n=0}^{\infty} \sum_{j} e_{j} \frac{(-)^{n}\left(\mathbf{r}_{j} \nabla\right)^{n}}{n !} \frac{1}{R} .
\end{aligned}
$$

При $R \gg r_{j}$ и $\sum_{j} e_{j}=0$ разложение (7) по степеням $1 / R$ начинается с дипольного потенциала

$$
\begin{gathered}
U(\mathbf{R})=-\sum_{j} e_{j} \mathbf{r}_{j} \nabla \frac{1}{R}+\ldots=\frac{\mathbf{D R}}{R^{3}}+\ldots, \\
\mathbf{D}=\sum_{j} e_{j} \mathbf{r}_{j} .
\end{gathered}
$$

Здесь D - дипольный момент увлекающей частицы, а $\mathbf{R}$ - расстояние между электроном и центром нейтральной частицы.

При $e_{j} \equiv 0$, например, для нейтрального атома, дипольный момент наводится зарядом электрона наноструктуры и выражается через поляризуемость $\mathbf{P}$ увлекающей частицы: $D_{\alpha}=P_{\alpha \beta} R_{\beta} / R^{3}$ с потенциалом взаимодействия $U(R)=P_{\alpha \beta} R_{\alpha} R_{\beta} / R^{6}$.

В силу закона сохранения (квази)импульса в формуле (6), матричный элемент потенциала взаимодействия оказывается фурье-образом потенциала $U(\mathbf{R})$ по переданному (квази)импульсу $\mathbf{q}=\mathbf{k}-\mathbf{p}=\mathbf{l}-\mathbf{l}^{\prime}$.

Вычислим фурье-образ потенциала $U(x, y, z)$ по координатам в плоскости $(x, y)$, считая координату $z$ (расстояние между увлекающей частицей и плоскостью наноструктуры) фиксированной. Для потенциала вида $D_{s}(x, y, z) / R^{s}$ имеем

$$
\Phi(\mathbf{q}, z)=\int d x d y \frac{D_{s}(x, y, z)}{\left(x^{2}+y^{2}+z^{2}\right)^{s / 2}} e^{i q_{x} x+i q_{y} y} .
$$

Поскольку координата $z$ всегда отлична от нуля, особенностей в нуле потенциал не имеет. На бесконечности интеграл (9) также сходится.

Функцию $\Phi(\mathbf{q}, z)$ можно выразить через более простой интеграл, содержащий только степени $1 / R$ :

$$
\Phi(\mathbf{q}, z)=\hat{D}_{s}(\mathbf{Q}) \Theta(\mathbf{q}, z) .
$$


Здесь

$$
\Theta\left(q_{x}, q_{y} \mid z\right)=\int d x d y \frac{e^{i q_{x} x+i q_{y} y}}{\left(x^{2}+y^{2}+z^{2}\right)^{s / 2}} .
$$

Операторная функция $\hat{D}_{s}(\mathbf{Q})$ от $\mathbf{Q}=-i \nabla_{q}$ связывает оба фурье-образа, так как $\hat{D}_{s}(\mathbf{Q}) \exp (i \mathbf{q r})=$ $=D_{s}(\mathbf{r}) \exp (i \mathbf{q r})$. Найдем $\Theta(\mathbf{q}, z)$ для произвольной степени $1 / R$. Это удобно сделать с помощью вспомогательного интегрирования, подняв знаменатель $R^{s}$ в показатель экспоненты:

$$
\frac{1}{\left(x^{2}+y^{2}+z^{2}\right)^{s / 2}}=\frac{1}{\Gamma(s / 2)} \int_{0}^{\infty} d t e^{-t\left(x^{2}+y^{2}+z^{2}\right)} t^{s / 2-1} .
$$

Формула (12) следует из интегрального представления для гамма-функции $\Gamma(s / 2)$. Далее, используя известные интегралы находим

$$
\int_{0}^{\infty} d x e^{-t x^{2}-i q_{x} x}=\sqrt{\frac{\pi}{4 t}} e^{-q_{x}^{2} / 4 t} .
$$

Аналогичный интеграл получится по оси $y$, так что формула для $\Theta(q, z)$ примет вид

$$
\Theta(q, z)=\frac{\pi}{4} \int_{0}^{\infty} d t e^{-t z^{2}-q^{2} / 4 t} t^{s / 2-2} .
$$

Теперь следует сделать масштабное преобразование для вспомогательной переменной $t \rightarrow c t$, чтобы привести интеграл (14) к табличному виду. Выбирая константу в виде $c=q / 2 z$, получаем

$$
\Theta(q, z)=\frac{\pi}{4}(q / 2 z)^{s / 2-1} \int_{0}^{\infty} d t e^{-q z / 2(t+1 / t)} t^{s / 2-2} .
$$

Если ввести новую переменную интегрирования $u$ по формуле $t=e^{u}$, то интеграл в (15) окажется удвоенным интегральным представлением функции Макдональда

$$
K_{v}(\xi)=\int_{0}^{\infty} d t e^{-\xi c h u} \operatorname{chv} \xi
$$

при $v=s / 2-1$ и $\xi=q z$.

Таким образом, фурье-образ функции вида $1 / R^{s}$ для $s=2 v+2$ оказывается равным:

$$
\Theta(q, z)=\frac{\pi}{2}(q / 2 z)^{v} K_{v}(q z) .
$$

Заметим, что асимптотика функций Макдональда для больших значений аргумента универсальна и не зависит от индекса $v: K_{v}(\xi) \simeq \sqrt{\pi / 2 \xi} e^{-\xi}$.

Дифференцируя (17) по компонентам вектора q можно найти фурье-образ $\Phi(\mathbf{q}, z)$ действующего потенциала вида (9) и затем вероятность перехода между электронными состояниям, пропорциональная квадрату этой величины. При $q z \geq 1$ он имеет вид степенной функции от $q$ и $z$ с экспоненциальным множителем $\exp (-2 q z)$.

Найдем ток увлечения для стационарного режима, при котором добавка к функции распределения равна

$$
\Delta F_{p}=-I_{p}^{-1} \Pi_{p}\left(F_{p}\right) .
$$

Подставляя это решение в формулу для тока (1) получаем ток увлечения

$$
J_{\alpha}^{d}=-e^{2} \sum_{p} v_{\alpha} I_{p}^{-1} \Pi_{p}\left(F_{p}\right) .
$$

В борновском приближении вероятности ухода и прихода в интеграле парных столкновений одинаковы $W_{k l^{\prime}}^{p l}=W_{p l}^{k l^{\prime}}$, кроме того, ввиду достаточной мягкости потенциала взаимодействия можно пренебречь обменным вкладом. Для $\Pi_{p}(F)$ в этом приближении имеем

$$
\Pi_{p}\left(F_{p}\right)=\sum_{k l l^{\prime}} W_{k p}^{l^{\prime} l}\left(F_{p} \Phi_{l}-F_{k} \Phi_{l^{\prime}}\right) .
$$

Из законов сохранения энергии и импульса в (6) с учетом только первого заполненного уровня поперечного квантования по оси $z$ следует условие для $x, y$ компонент скоростей электронов наноструктуры $v_{x}, v_{y}$, увлекающих частиц $V \equiv V_{x}$ и передаваемого импульса $q$

$$
q^{2}(1 / 2 M+1 / 2 m)=q_{x}\left(v_{x}-V_{x}\right)+q_{y} v_{y} .
$$

Поскольку имеется очень большая разницу масс электронов $m$ и внешних частиц $M$, весь процесс взаимодействия можно представить как почти упругие столкновения электронов проводимости с движущимися с постоянной скоростью $V=V_{x}$ массивными рассеивателями. Ввиду малости отношения $m / M \ll 1$ скорость и энергия массивной частицы мало меняется и условие сохранения энергии-импульса сводится к равенству $q^{2} / 2 m=\left[q_{x}\left(V-v_{x}\right)-q_{y} v_{y}\right]$, что позволяет упростить выражение для $\Pi_{p}\left(F_{p}\right)$ :

$$
\begin{aligned}
\Pi_{p}\left(F_{p}\right) & =\sum_{q l} W_{p+q l-q}^{p l}\left(F_{p} \Phi_{l}-F_{p+q} \Phi_{l-q}\right) \\
& \simeq \sum_{q_{x} q_{y}} w_{q}(p, V) N_{V}\left(F_{p}-F_{p+q}\right) .
\end{aligned}
$$

Здесь $N_{V}=\Phi_{l} \simeq \Phi_{l-q}-$ число увлекающих частиц с данной скоростью $V$. Вероятность $w_{q}(p, V)$ передачи импульса $q$ электрону с импульсом $p$ равна

$$
\begin{aligned}
w_{q}(p, V)= & \frac{1}{(2 \pi)^{2}} \int d q_{x} d q_{y} \Phi^{2}(q z) \\
& \times \delta\left(q^{2} / 2 m+q_{x} v_{x}+q_{y} v_{y}-q_{x} V\right) .
\end{aligned}
$$

Если скорость потока увлекающих частиц невелика по сравнению со средней скоростью электронов, то действие оператора $\Pi_{p}\left(F_{p}\right)$ можно представить как плавное 
изменение распределения электронов $F_{p}$ под действием „силы увлечения“

$$
\Pi_{p}\left(F_{p}\right) \simeq \sum_{q_{x} q_{y}} w_{q}^{p, V} q_{\beta} \frac{\partial F_{p}}{\partial p_{\beta}}=f_{\beta}^{d}(p) \frac{\partial F_{p}}{\partial p_{\beta}} .
$$

Эта сила выражается через отношения переданного (квази)импульса к интервалу времени между столкновениями электронов с увлекающими их частицами, что можно условно записать в обычном для силы виде как $f^{d}=q / \tau_{d}=\Delta \dot{p}$.

Можно ввести также среднее поле увлечения, приравнивая ток увлечения эквивалентному омическому току

$$
J_{\alpha}^{d}=-e^{2} \sum_{p} v_{\alpha} I_{p}^{-1} \frac{\partial F}{\partial p_{\beta}} f_{\beta}^{d}(p)=\sigma_{\alpha \beta} E_{\beta}^{d} .
$$

Здесь $\sigma_{\alpha \beta}$ - тензор проводимости образца.

При усреднении по ориентации нейтральных молекул их дипольный потенциал в среднем исчезает (см. [10]), так что следует учитывать поляризацию при действии кулоновского потенциала электрона. В итоге сила увлечения определяется поляризуемостью увлекающих частиц и их средним расстоянием от наноструктуры. Она экспоненциально убывает при увеличении этого расстояния как $\exp (-2 q z)$. Для тяжелых увлекающих частиц изменение (квази)импульса электронов имеет порядок величины $q \sim m V^{\prime}<m V$. Для $m V z \geq 1$ порядок величины увлечения слабо зависит от формы действующего потенциала и для его оценки можно использовать данные работ $[8,9]$.

\section{Список литературы}

[1] М.Б. Погребинский. ФТП 11, 637, (1977).

[2] V.L. Gurevich, V.B. Pevzner, E.W. Fenton. J. Phys.: Condens. Matter 10, 2551 (1998).

[3] J.S. Moon, M.A. Blount, J. Simmons, J.R. Wendt, S.K. Lyo, J.L. Reno. Phys. Rev. B 60, 11530 (1999).

[4] P. Debray, P. Vasilopoulos, O. Raichev, R. Perrin, M. Rahman, W.C. Mitchel. Physica E 6, 694 (2000).

[5] P. Debray, V. Zverev, O. Raichev, R. Klesse, P. Vasilopoulos, R.S. Newrock. J. Phys.: Condens. Matter 13, 3389 (2001).

[6] V.L. Gurevich, M.I. Muradov. Zh. Eksp. Teor. Fiz. Pis'ma Red. 71, 164 (2000).

[7] V.L. Gurevich, M.I. Muradov. J. Phys.:Condens. Matter 14, 3300 (2001).

[8] V.L. Gurevich, M.I. Muradov. ЖЭТФ 148, 1137 (2015).

[9] В.Л. Гуревич, М.И. Мурадов. ФТТ 57, 12, 2429 (2015).

[10] С.В. Ганцевич, В.Л. Гуревич, Е.М. Собко, ФТТ 49, 12, 2125 (2007).

Редактор Т.Н. Василевская 